\title{
Completeness of primary intracranial tumour recording in the Scottish Cancer Registry
}

2011-12

Authors:

Joanne R. Morling ${ }^{\mathrm{a}}$, Robin Grant ${ }^{\mathrm{b}}$ and David H. Brewster ${ }^{\mathrm{a}}$.

a. Information Services Division, NHS National Services Scotland, 1 Gyle Square, South Gyle Crescent, Edinburgh, EH12 9EB, UK

b. Department of Neurosciences, Western General Hospital, Crewe Road South, Edinburgh, EH4 2XU, UK

Corresponding author:

Dr Joanne Morling

Division of Epidemiology and Public Health, University of Nottingham, City Hospital Campus, Hucknall Road, Nottingham NG5 1PB.

Tel. +44 (0)1158231812

Email: joanne.morling@nottingham.ac.uk 
Keywords: brain neoplasms; data collection; glioma; medical record linkage; quality control; registries

\section{INTRODUCTION}

A high level of case ascertainment by cancer registries is essential to allow estimation of accurate incidence rates and survival. Nearly 20 years ago, researchers assessed the completeness and accuracy of registration of primary intracranial tumours in the Scottish Cancer Registry (SCR) ${ }^{1}$ compared to a database assembled in the context of a detailed incidence study carried out in the Lothian region of Scotland covering the period of diagnosis, 1989-1990 $0^{1-2}$. Disappointingly, SCR identified only $54 \%$ of cases, although the registry at that time did not attempt to collect information on 'benign' intracranial neoplasms which were included in the detailed incidence study. Even so, only $84 \%$ of neuro-epithelial tumours were identified by SCR, probably related in part to the fact that, the cancer registry was not receiving neuropathology data from the regional neuro-oncology centre. An English study reported similar findings with only 52\% of cases appearing in the regional cancer registry ${ }^{3}$.

Over time, access to diagnostic techniques has improved alongside improvements and changes in classification and clinical coding. Furthermore, SCR now receives neuropathology data from all neuro-oncology centres in Scotland, and has sought to collect information on benign tumours of the brain and spinal cord since the year of diagnosis, 2000. In light of these developments, we aimed to determine the completeness of ascertainment of primary intracranial tumours by SCR through independent/clinical case ascertainment in NHS Lothian for the period of diagnosis, 2011-2012.

\footnotetext{
${ }^{1}$ ICD-O-3 International Classification of Diseases for Oncology; MDTM multidisciplinary team meeting; National Records Scotland; SCR Scottish Cancer Registry; SMR01 Scottish Morbidity Record 01; SNOMED Systematized Nomenclature of Medicine.
} 


\section{METHODS}

\section{Scottish Cancer Registry (SCR)}

SCR operates by bringing together predominantly electronic information from: hospital patient administration systems including patient discharges from hospital (SMR01), radiotherapy, oncology, pathology records; death records from National Records Scotland (NRS); and private hospital records ${ }^{4}$. All primary malignant and benign brain tumours are recorded on the SCR.

\section{Inclusion and exclusion criteria}

This retrospective cohort study was restricted to the period of diagnosis between $1 / 1 / 11$ and $31 / 12 / 12$ and limited to adults (age $\geq 18$ years on the date of diagnosis) with a postcode within the NHS Lothian health board region (mid-year population 650,000). The date of diagnosis was taken as i) the date of the first abnormal imaging, or ii) the date of biopsy/resection. Patients in whom there was no neuro-radiology or histology were excluded, i.e. diagnosis of prolactinoma had to be supported by blood tests and imaging.

All suspected and histologically proven primary intracranial tumours (benign and malignant) of the brain and cranial nerves were counted, including primary central nervous system lymphoma. Meningeal, pituitary region and pituitary gland tumours were also included. Cerebral metastases, tumours of the spinal cord and spinal nerves, and recurrent intracranial tumours of any type were excluded.

\section{Extraction from the SCR}

Data were extracted for the study period for all records including the following anatomical site codes selected from the third edition of the International Classification of Diseases for Oncology (ICD-O-3): C70.0, C70.9, C71, C72.2, C72.3, C72.4, C72.5, C72.8, C72.9, C75.1, C75.2, and C75.3 (all behaviour codes). 


\section{Clinical case ascertainment}

Three clinical sources were trawled: i) neuro-oncology multidisciplinary meeting minutes (MTDM); ii) an endocrinology database; and iii) neuropathology records (sources $\mathrm{i}$ and ii are independent of the SCR data collection system). The neuro-oncology MDTM aims to discuss all intracranial tumours identified via any means including both benign and malignant tumours. The endocrinology database records all patients attending the endocrine outpatient clinics in the NHS Lothian region and each is assigned a diagnosis by a Consultant Endocrinologist. Both sources $\mathrm{i}$ and ii for the period 1 Jan 2011 to 31 March 2013 were reviewed manually by JRM. The neuropathology records hold information on all tissue samples analysed in the pathology system for NHS Lothian hospitals and an electronic extract was obtained using Systematized Nomenclature of Medicine (SNOMED) codes matching those above. To ensure cases were true incident cases meeting the full inclusion criteria, each was cross referenced with the patient's electronic secondary care medical record.

\section{Analysis}

Clinically ascertained cases were reconciled against the SCR extract. We have previously quantified the extent of misclassification of incidence year in the Scottish Cancer Registration database ${ }^{5}$. We did not regard misclassifications of incidence year as missed or 'over-diagnosed' cases as there is no reason to believe that such misclassification is other than random.

Completeness was defined as the proportion of intracranial tumours included in the SCR out of all those identified as diagnosed in residents of NHS Lothian area during the study period. Confidence intervals (95\%) for completeness were calculated using the exact method.

Completeness was calculated for all intracranial tumours with secondary analysis of completeness by tumour morphology and tumour grade (see Supplementary File for coding definitions). 


\section{Ethical approval}

The study was considered health-service audit and did not require NHS ethical review as the project was limited to the utilisation of data previously collected in the course of normal care. NHS Lothian Caldicott Guardian approval was obtained for the use of person-identifiable information.

\section{RESULTS}

There were 320 records of primary intracranial tumours registered on the SCR for the period of interest and 264 clinical cases were ascertained. Figure 1 shows the final ascertainment of clinical cases missing from the SCR.

42 clinical cases were identified as missing from the SCR, with 98 cases on the SCR not identified by searching clinical sources. The 42 missing cases were identified from: neuro-oncology MDTM (7), endocrinology out-patient database (30) and neuropathology records (5).

Overall case ascertainment was $88.4 \%$, which after exclusion of pituitary adenomas ( $22.6 \%$ macroand $77.4 \%$ micro-adenomas) rose to $96.3 \%$

Table 1 shows the ascertainment by tumour morphology. All tumour types had ascertainment $>90 \%$ with the exception of pituitary adenomas (53.7\%) and unidentifiable tumours (77.8\%). Considering tumours by grade (with pituitary adenomas removed due to poor acquisition), missing cases were most prominent in the high grade (IV) tumours, with $4.8 \%$ of cases missing.

Of the 98 cases on the SCR not identified in the clinical case ascertainment the reasons for nonascertainment were: $7(7.1 \%)$ died within 1-month of the diagnosis or were registered from death certification/autopsy, 9 (9.2\%) had a diagnostic date recorded in their medical records outside of the period 2011-12, 79 (80.6\%) were confirmed as accurate registrations from their medical records but 
were not referred to the neuro-oncology MDTM or had pathology specimens taken, and 3 (3.1\%) were diagnosed and/or treated outside of the NHS Lothian region.

The morphology of these 98 were: 9 (9.2\%) neuroepithelial, 19 (19.4\%) cranial nerve, 49 (50.0\%) meningeal, 1 (1.0\%) primary CNS lymphoma, 11 (11.2\%) pituitary adenomas, and 9 (9.2\%) unknown.

\section{DISCUSSION}

This study found the SCR to be near complete (96.3\%) for non-pituitary intracranial tumours. This finding is reassuring given the increasing reliance on routine data sources in clinical research in the 'big data' era.

A key strength of this work was the use of predominantly independent clinical case ascertainment. Two of the three sources used were independent of the SCR with the pathology database part of the routine reporting system. Use of dependent sources would lean to an over-estimation of the completeness, however, we are confident in our finding as the search employed for the pathology extract was more extensive than the SCR compiling extract and we did identify further missing cases. To further augment the clinical case ascertainment imaging sources could also be used. Unfortunately it was not possible for us to do this due to the very high volume of imaging activity and the fact that report findings are not synoptic or coded.

Another possible weakness was the use of a single researcher (JRM) in identifying independent cases. To try and minimise missing cases or misclassification, all missing cases had the medical records rechecked by the researcher and a member of the SCR data quality assurance team reviewed the registry for the entry, outside of the extract requested.

This work replaces prior completeness estimates published in the late $1990^{\prime} \mathrm{s}^{1}$ and shows a clear improvement in the completeness of ascertainment. This is likely to relate to operational changes in the way that data are collected by SCR and the decision to collect data on benign brain tumours 
from year 2000 onwards. It is possible that completeness of ascertainment of tumours varies by site with other cancers potentially having higher completeness, however these findings are more consistent with estimates of completeness of ascertainment for other cancer sites/types in $\mathrm{SCR}^{6-7}$.

It is important that outdated estimations of completeness of ascertainment of intracranial tumours are revised to ensure robust estimates of intracranial tumour incidence are made and understood. The use of outdated information can lead to misleading inferences, for example, The Brain Tumour Charity continues to report a belief that "many brain tumours are not recorded" in official statistics in Scotland ${ }^{8}$.

\section{Conclusion}

Given the increase in intracranial tumours being diagnosed by imaging we would recommend a move towards diagnostic coding of imaging in Scotland. A further issue to address in the future relates to the recording of benign pituitary tumours - predominantly microadenomas. We found that most of these were noted in the records of out-patient endocrinology attendances following $\mathrm{CT} / \mathrm{MRI}$ scanning. It is unclear how a national system to collect these could be implemented because, at present, diagnosis is not coded in either computerised imaging data or outpatient data collected routinely.

[words 1538] 


\section{ACKNOWLEDGEMENTS}

We are grateful to, Dr F. Gibb, A. Henriquez, Prof J. Ironside, K. Lake, A. MacDonald, I. MacDonald, Dr M. Strachan and S. Young for assistance with, accessing and validating, the SCR and clinical case records. We also thank the Edinburgh Clinical Neuro-Onocology Multidisciplinary Team for access to and assistance with the multidisciplinary team meeting minutes.

\section{Ethical approval}

The study was considered health-service audit and did not require NHS ethical review as the project was limited to the utilisation of data previously collected in the course of normal care. NHS Lothian Caldicott Guardian approval was obtained for the use of person-identifiable information.

\section{Funding}

No funding.

\section{Conflicts of interest}

JRM is an Associate Editor of the journal Public Health

RG and DHB have no conflict of interest to declare.

\section{Authorship}

J.R.M. designed the study, collected the data, analysed the data, wrote the manuscript and approved the final version. R.G. assisted with study design, assisted with data collection, revised the manuscript and approved the final version. D.H.B. designed the study, assisted with data analysis, revised the manuscript and approved the final version.

No assistance was received in the writing or editing of this manuscript from any persons who are not named as authors of the manuscript. 


\section{REFERENCES}

1. Counsell CE, Collie DA, Grant R. Incidence of intracranial tumours in the Lothian region of Scotland, 1989-90. J Neurol Neurosurg Psychiatry 1996;61:143-50.

2. Counsell CE, Collie DA, Grant R. Limitations of using a cancer registry to identify incident primary intracranial tumours. J Neurol Neurosurg Psychiatry 1997;63:94-7.

3. Pobereskin L. The completeness of brain tumour registration in Devon and Cornwall. Eur $J$ Epidemiol 2001;17:413-6.

4. Brewster DH, Kerssens J, Bhatti L, et al. United Kingdom, Scotland. In: Forman D, Bray F, Brewster DH, Gombe Mbalawa C, Kohler B, Piñeros M, Steliarova-Foucher E, Swaminathan R, Ferlay J, editors. Cancer Incidence in Five Continents, Vol X, IARC Scientific Publications No. 164, Lyon, France, 2014, p 878.

5. Brewster D, Crichton J, Muir CS. How accurate are Scottish cancer registration data? Br J Cancer 1994;70:954-959.

6. Brewster D, Crichton J, Harvey JC, et al. Completeness of case ascertainment in a Scottish Regional Cancer Registry for the year 1992. Public Health 1997;111:339-343.

7. Brewster DH, Stockton DL. Ascertainment of breast cancer by the Scottish Cancer Registry: An assessment based on comparison with five independent breast cancer trials databases. Breast 2008;17:106-108.

8. The Brain Tumour Charity http://www.thebraintumourcharity.org/Scotland/The-facts accessed August 2015. 


\section{TABLES AND FIGURES}

Figure 1 Flowchart of ascertainment of cases missing from the Scottish Cancer Registry (SCR).

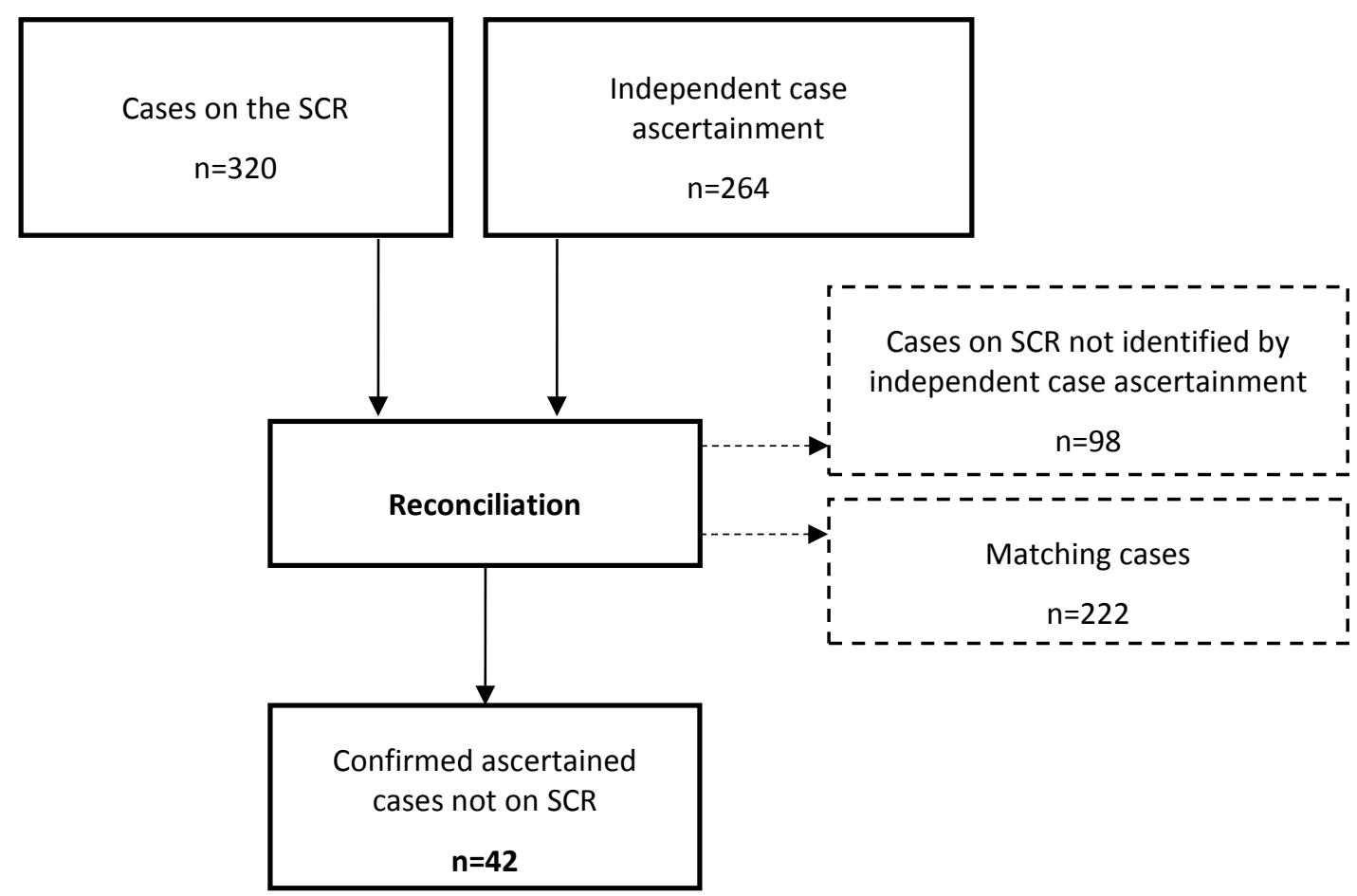

SCR Scottish Cancer Registry 
Table 1 Completeness of primary intracranial tumour recording on the Scottish Cancer Registry (SCR) for NHS Lothian by tumour morphology

\begin{tabular}{|c|c|c|c|c|}
\hline Tumour morphology & $\begin{array}{l}\text { Number on } \\
\text { SCR [a] }\end{array}$ & $\begin{array}{c}\text { Additional } \\
\text { 'clinical' cases } \\
\text { [b] } \\
\end{array}$ & $\begin{array}{c}\text { Case ascertainment, } \\
\%(95 \% \mathrm{Cl})[\mathrm{a} / \mathrm{a}+\mathrm{b}]\end{array}$ & $\begin{array}{c}\text { Crude incidence ( } \geq 18 \\
\text { years) per } 100,000 \\
\text { person-years }\end{array}$ \\
\hline Neuroepithelial & 134 & 5 & $\begin{array}{c}96.4 \\
(91.4,98.7)\end{array}$ & 10.6 \\
\hline Cranial nerves & 22 & 1 & $\begin{array}{c}95.7 \\
(76.0,99.8)\end{array}$ & 1.8 \\
\hline Tumours of the meninges & 105 & 1 & $\begin{array}{c}99.1 \\
(94.1,100)\end{array}$ & 8.1 \\
\hline $\begin{array}{l}\text { Lymphomas and } \\
\text { haemopoetic }\end{array}$ & 7 & 0 & $\begin{array}{c}100 \\
(56.1,100)\end{array}$ & 0.5 \\
\hline Germ cell & 0 & 0 & - & - \\
\hline Sellar region & 2 & 0 & $\begin{array}{c}100 \\
(19.8,100)\end{array}$ & 0.2 \\
\hline $\begin{array}{l}\text { Pituitary } \\
\text { adenoma/prolactinoma }\end{array}$ & 36 & 31 & $\begin{array}{c}53.7 \\
(41.2,65.8)\end{array}$ & 4.9 \\
\hline Unknown & 14 & 4 & $\begin{array}{c}77.8 \\
(51.9,92.6) \\
\end{array}$ & 1.4 \\
\hline Total & 320 & 42 & $\begin{array}{c}88.4 \\
(84.5,91.4)\end{array}$ & 27.6 \\
\hline Excluding pituitary tumours & 284 & 11 & $\begin{array}{c}96.3 \\
(93.2-98.0)\end{array}$ & 22.5 \\
\hline
\end{tabular}

